\title{
Graphical-analytical analysis of the mechanism with rotating cam and flat- face follower
}

\author{
Stelian Alaci ${ }^{1, *}$, Florina-Carmen Ciornei $^{1}$, Sorinel-Toderas Siretean ${ }^{1}$ and Mariana-Catalina Ciornei $^{2}$ \\ ${ }^{1}$ Stefan cel Mare University, Suceava, 13 University Str., 720229, Romania \\ ${ }^{2}$ Carol Davila University of Medicine and Pharmacy, Dpt. Clinic 2, 8 Bd. Eroii Sanitari, 050474, Bucharest, Romania
}

\begin{abstract}
The graphical-analytical methods are mentioned as being straightforward and expedite. The paper presents a graphical-analytical method for the kinematical analysis of a mechanism with rotating cam and oscillating flat-face follower. The manner supposes considering the contact point as a separate element of the mechanism and using concurrently the replacing mechanism with lower pairs and the actual mechanism, the relative motions from the contact point of the cam and contact point of the follower, respectively, are established. The methodology is exemplified for an actual case.
\end{abstract}

\section{Introduction}

The cam mechanisms are identified taking into account the motion of the cam, the motion of the follower and the type of contact between the cam and the follower [1]. Considering the last criterion, the followers are of knifeedge type, when a single contact point exists with the cam, and from flat-face category, when the follower is tangent permanently to the profile of the cam. The kinematical study mechanisms can be made applying graphical, graphical-analytical or analytical procedures. The graphical-analytical methodologies have as foremost advantages the straightforward mathematical apparatus and quickness of solving. The main drawback of the method consists in the fact that it can be applied for a specified position of the cam. This inconvenience can be eliminated by the analytical methods. The disadvantage of the analytical methods resides in the complex mathematical apparatus, [2-3].

\section{General considerations upon cam mechanisms}

The graphical-analytical kinematics of a cam mechanism requires as principle, establishing the analytical expressions for the velocity/acceleration of a point of the mechanism (for the actual case, the contact point) in two manners, and afterwards solving graphically these equations by means of the velocities and accelerations polygons. In the case of the mechanisms with flat face follower, these systems are undetermined systems (as it will be proved later). An artifice is presented in [4] for overcoming this drawback, that is using a fictive follower with the knife edge placed in the cam-follower contact point. The fictive follower creates two mechanisms, the first one with the cam and the second one with the follower. The constraint imposed to the fictive follower, to have the same motion in both mechanisms, conducts to the absolute motion of the follower and the motion from the higher pair. Pelecudi [5] remarks that there are several equivalent mechanisms for the cam mechanism and proposes for solving the problem the selection of a certain mechanism. A method for the kinematical graphicalanalytical analysis was propose and applied in a recent paper [6] for a mechanism with cam and curved follower with non-zero curvature. In the paper it is noticed that the method cannot be applied for mechanisms with rotating cam and flat face follower, Fig. 1, since the two mechanisms are not structurally equivalent. For this reason, a method for kinematical analysis of the mechanism with flat face follower is presented next with emphasis upon the causes impeding the direct establishing of the motion of the follower and of the relative camfollower motion.

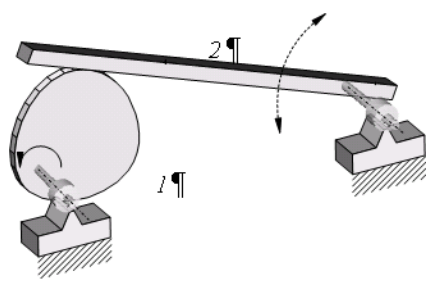

Fig. 1. Mechanism with rotating cam and oscillating flat face follower

To this end, the mechanisms with oscillating flat-face follower from Fig. 2 is considered, where the cam is made from a disc with $R$ radius mounted eccentrically at distance $\delta$ with respect to the centre. The equivalent mechanism with plane pairs is also shown in Fig. 2. The vector contour from Fig. 3 is considered and the vector equation of closed vector contour is:

$$
\boldsymbol{L}_{1}+\boldsymbol{L}_{2}+\boldsymbol{L}_{3}+\boldsymbol{L}_{0}=\mathbf{0}
$$

\footnotetext{
* Corresponding author: alaci@fim.usv.ro
} 


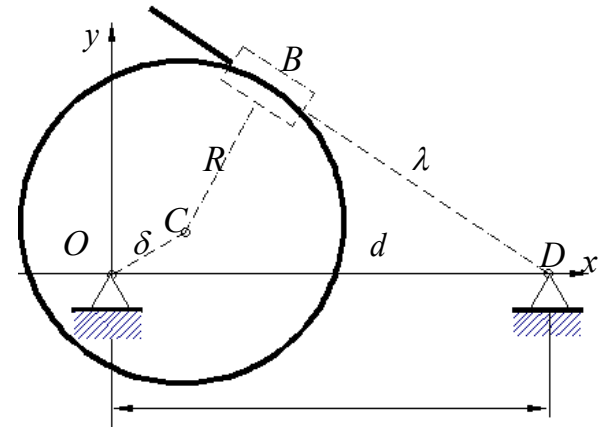

Fig. 2. Circular eccentric with oscillating flat-face follower

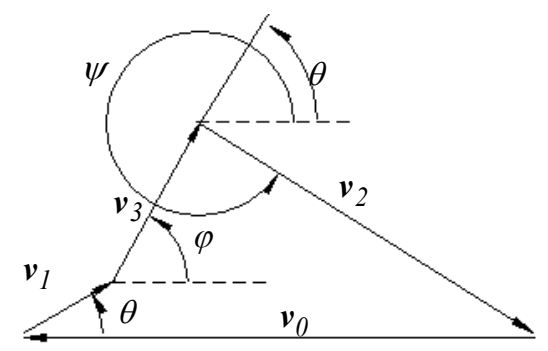

Fig. 3. The equivalent mechanism of the cam mechanism

and has the equations of projections:

$$
\left\{\begin{array}{l}
\delta \cos \theta+R \cos \varphi+\lambda \cos \psi-d=0 \\
\delta \sin \theta+R \sin \varphi+\lambda \sin \psi=0
\end{array}\right.
$$

with $\varphi, \psi$ and $\psi$ as unknowns. Adding the equation:

$$
\psi=\varphi+3 \pi / 2
$$

the system becomes compatible and has the solutions:

$$
\varphi_{1,2}=2 \operatorname{atan} \frac{d \sin \theta \pm \sqrt{\delta^{2}-R^{2}-2 \delta d \cos \delta+\delta^{2}}}{\delta \cos R-d-R}
$$

In Fig. 4 there are presented the cam and the follower occupying the two positions and afterwards, using the relations:

$$
\begin{aligned}
& x_{C_{1,2}}=\delta \cos \theta+R \cos \varphi_{1,2} \\
& y_{C_{1,2}}=\delta \sin \varphi+R \sin \varphi_{1,2}
\end{aligned}
$$

the two trajectories of the contact points from the two mechanisms were traces. There were also represented two knife-edge followers having the same joint $D$ with the ground having the knife-edges in the two contact points. From this representation one can notice the essential difference between the two mechanisms. While for the knife-edge follower the contact point is immobile on the follower, the distance $\lambda$ being constant, and the absolute trajectory is represented by the circles $\Gamma_{1,2}$ with the centers in the joint $D$, for the case of the mechanisms with plat-face follower, the contact point is mobile both on the cam and on the follower. As well, the trajectories of the contact points are two unknown curves and therefore, for a given position of the cam, the directions of the absolute velocities $v_{C_{12}}$ of the contact points are not known, compared to the case of the knife-edge follower, when the velocity of the contact point is always normal to the direction of the follower.

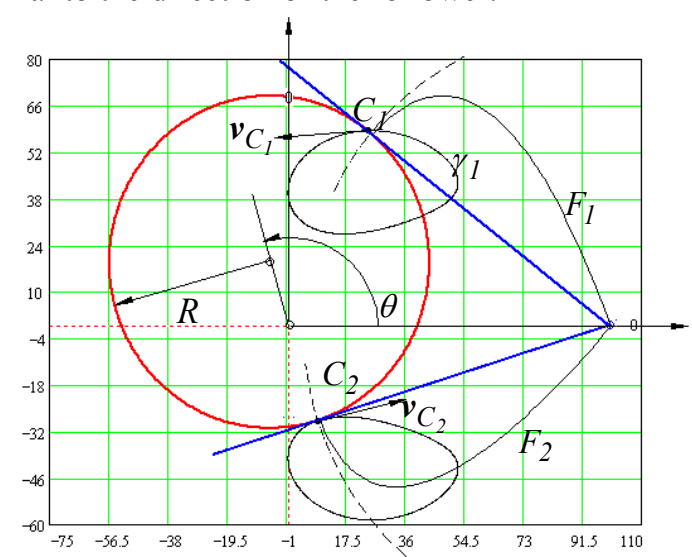

Fig. 4. The cam and the follower occupying the two positions corresponding to the solutions

From here it results the conclusion that three unknowns occur in the system of plan vector equations that expresses the absolute velocity of the contact point: the absolute motion of the follower and the relative motions of the contact point with respect to the cam and to the follower.

\section{The kinematical graphical-analytical analysis of the mechanism}

The motion of a point $B_{2}$ on a mobile plane curve is considered and it is assumed that at the moment of the analysis the point $B_{2}$ overlies on the $B_{1}$ point placed on the curve. The following relations are valid between the velocities of the two points:

$$
\boldsymbol{v}_{B_{2}}=\underline{\underline{v_{B_{1}}}}+\frac{v_{B_{2} B_{1}}}{\| t t}
$$

and between the accelerations:

$$
\boldsymbol{a}_{B_{2}}=\underline{\underline{\boldsymbol{a}_{B_{1}}}}+\frac{\boldsymbol{a}_{B_{2} B_{1}}^{t n}}{\| n n}+\frac{\boldsymbol{a}_{B_{2} B_{1}}^{c}}{\| n n}+\frac{\boldsymbol{a}_{B_{2} B_{I}}^{t n}}{\| t t}
$$

The corresponding equation in the polygon of velocities:

$$
\overline{p_{v} b_{2}}=\overline{p_{v} b_{1}}+\overline{b_{2} b_{1}}
$$

and in the polygon of accelerations, respectively:

$$
\overline{p_{a} b_{2}^{\prime}}=\overline{p_{a} b_{1}^{\prime}}+\overline{b_{1}^{\prime} n_{B_{2} B_{1}}}+\overline{n_{B_{2} B_{1}} k_{B_{2} B_{1}}+\overline{k_{B_{2} B_{1}} b_{2}^{\prime}}}
$$

In the above relations, $n n$ and $t t$ are the notations for the directions parallel to the normal and tangent in the contact point, respectively. 


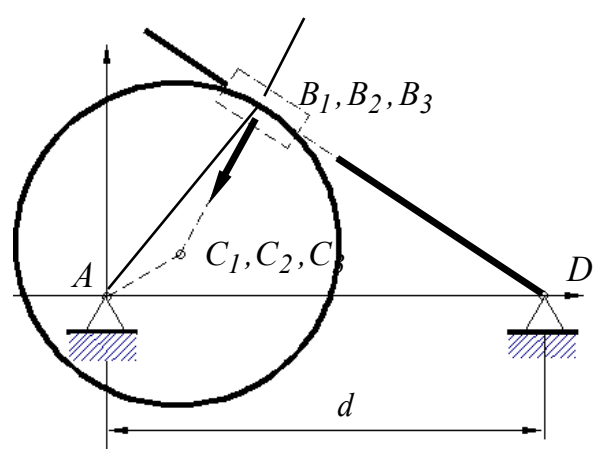

Fig. 5. The actual mechanism and the replacing mechanism

The normal transport acceleration $\boldsymbol{a}_{B_{2} B_{1}}^{t n}$ is defined by the relation:

$$
\boldsymbol{a}_{B_{2} B_{1}}^{t n}=-\left(v_{B_{2} B_{1}}^{2} / \rho\right) v
$$

where $\rho$ is the curvature radius and $\boldsymbol{n}$ is the versor of the normal. The relative Coriolis acceleration is:

$$
\boldsymbol{a}_{B_{2} B_{1}}^{c}=2 \omega_{1} \times \boldsymbol{v}_{B_{2} B_{1}}
$$

where $\omega_{1}$ is the angular velocity of the plane containing the curve. The relative acceleration is $\boldsymbol{a}_{B_{2} B_{1}}^{r}$, directed about the tangent in the contact point. Both the actual mechanism and the replacing mechanism are presented in Fig. 5. The element 3 replaces the higher pair and has at the ends a rotation joint placed in the centre of curvature of the cam and a prismatic joint in the contact point. In the centre of curvature of the cam and in the contact point there are the points $C_{1}, C_{2}, C_{3}$ and $B_{1}, B_{2}, B_{3}$ placed on the cam, on the follower and on the replacing element, respectively. Applying the relation 5 it is obtained, for the points $B_{3}$ and $B_{1}$ :

$$
\begin{aligned}
& \boldsymbol{v}_{B_{3}}=\underline{\underline{\boldsymbol{v}_{B_{1}}}}+\underline{\boldsymbol{v}_{B_{3} B_{1}}} \\
& \boldsymbol{v}_{B_{3}}=\underline{\underline{\boldsymbol{v}_{B_{2}}}}+\underline{\boldsymbol{v}_{B_{3} B_{2}}}
\end{aligned}
$$

and for the points $B_{3}$ and $B_{2}$, respectively:

$$
\begin{aligned}
& \boldsymbol{a}_{B_{3}}=\underline{\underline{\boldsymbol{a}_{B_{1}}}}+\underline{\boldsymbol{a}_{B_{3} B_{1}}^{t n}}+\underline{\boldsymbol{a}_{B_{3} B_{1}}^{c}}+\underline{\boldsymbol{a}_{B_{3} B_{1}}^{t n}} \\
& \boldsymbol{a}_{B_{3}}=\underline{\underline{\boldsymbol{a}_{B_{2}}}}+\underline{\boldsymbol{a}_{B_{3} B_{2}}^{t n}}+\underline{\boldsymbol{a}_{B_{3} B_{2}}^{c}}+\underline{\boldsymbol{a}_{B_{3} B_{2}}^{t n}}
\end{aligned}
$$

Concerning the velocities, the directions of the relative velocities $\boldsymbol{v}_{B_{3} B_{1}}, \boldsymbol{v}_{B_{3} B_{2}}$ are unknown but this can be overcome by subtracting the equations 12 , member by member and writing the results under the form:

$$
\boldsymbol{v}_{B_{2}}=\boldsymbol{v}_{B_{1}}+\boldsymbol{v}_{B_{2} B_{1}}
$$

In the equation obtained above, $\boldsymbol{v}_{B_{I}}$ is completely known and the other two vectors have known directions, $\boldsymbol{v}_{B_{2}} \perp D B$ and $\boldsymbol{v}_{B_{2} B_{1}} \| t t(B D)$; therefore the equation allows for finding the velocity of the point $B_{2}$ and implicitly the angular velocity of the follower and the relative velocity from the higher pair. The challenge of applying the same methodology for the analysis of the accelerations reaches the barrier that there are necessary the relative velocities of the contact point with respect to the cam and to the follower; these velocities must be known for finding the magnitudes of the accelerations $\boldsymbol{a}_{B_{3} B_{1}}^{t n}, \boldsymbol{a}_{B_{3} B_{1}}^{c}$ and $\boldsymbol{a}_{B_{3} B_{1}}^{c}$; the acceleration $\boldsymbol{a}_{B_{3} B_{2}}^{t n}$ is zero since the curvature radius of the follower is $\rho_{2} \equiv \infty$. To surmount this difficulty it is recalled that due to the prismatic joint from point $B$ the next equality is always valid:

$$
\boldsymbol{v}_{B_{3} B_{2}}=\boldsymbol{v}_{C_{3} C_{2}}
$$

In the velocity polygon, the relation 15 is written as:

$$
\overline{b_{2} b_{3}}=\overline{c_{2} c_{3}}
$$

Moreover, it is taken into consideration that due to the revolution joint from $C, v_{C_{1}} \equiv v_{C_{3}}$ and the points $c_{1}$ and $c_{3}$ are always overlapped in the velocity polygon. The position of the point $c_{1}$ is found by applying the theorem of similar triangles between the known $A B C$ and $a b_{1} c_{1}$. Now, the point $c_{2}$ can be found by translation of the point $c_{3}$ with the vector $b_{3} b_{2}$. For establishing the accelerations distribution, the equation 4 is applied twice, for the points $B_{3}$ and $B_{1}$, and for $B_{3}$ and $B_{2}$ respectively:

$$
\left\{\begin{array}{l}
\bar{a}_{B_{3}}=\underline{\underline{\bar{a}_{B_{1} A}^{n}}}+\underline{\underline{\bar{a}_{B_{1} A}^{t}}}+\underline{\underline{\bar{a}_{B_{3} B_{1}}^{c}}}+\underline{\underline{\underline{a_{B_{3} B_{1}}}}}+\frac{+\bar{a}_{B_{3} B_{1}}^{t}}{\| t t} \\
\bar{a}_{B_{3}}=\underline{\bar{a}_{B_{2} D}^{t}+\bar{a}_{B_{2} D}^{n}}+\underline{\underline{\bar{a}_{B_{3} B_{2}}^{c}}}+\underline{\underline{a_{B_{3} B_{2}}^{t n}}}+\frac{\bar{a}_{B B_{2}}^{t}}{\| t t}
\end{array}\right.
$$

The right members of the equations 17 are equalled and introducing the notations:

$$
\begin{gathered}
\bar{a}_{B_{2} B_{1}}^{c}=\underline{\underline{\bar{a}_{B_{3} B_{1}}^{c}}}-\underline{\underline{\bar{a}_{B_{3} B_{2}}^{c}}} \\
\bar{a}_{B_{2} B_{1}}^{\text {tn }}=\underline{\underline{\bar{a}_{B_{3} B_{1}}^{t n}}}
\end{gathered}
$$

it results the following relation:

$$
\bar{a}_{B_{3} B_{1}}^{t}-\bar{a}_{B_{3} B_{2}}^{t}=\left(\bar{a}_{B_{3}}^{t}-\bar{a}_{B_{1}}^{t}\right)+\left(\bar{a}_{B}^{t}-\bar{a}_{B_{2}}^{t}\right)=\bar{a}_{B_{2} B_{1}}^{t}
$$

formally rewritten as: 


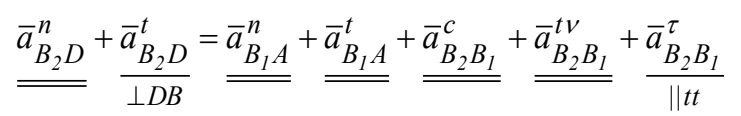

The above equation is formally identical to the equation of accelerations corresponding to the knife-edge follower with the remark that $\bar{a}_{B_{2} B_{I}}^{c}, \bar{a}_{B_{2} B_{I}}^{n \tau}$ are just notations and have different relations compared to the ones known for the two terms. The consequent equation from the polygon of accelerations is:

$$
\begin{aligned}
& {\overline{p_{a} n}}_{B_{2} D}+\overline{n_{B_{2} D} b_{2}^{\prime}}={\overline{p_{a} n}}_{B_{1} A}+ \\
& \overline{n_{B_{1} A b_{1}^{\prime}}}+{\overline{b_{1}^{\prime} k_{B_{2} B_{1}}}}+{\overline{n_{B_{2} B_{I}} b^{\prime}}}_{2}
\end{aligned}
$$

The solving of the equation in the accelerations' polygon is presented in Fig. 6 .
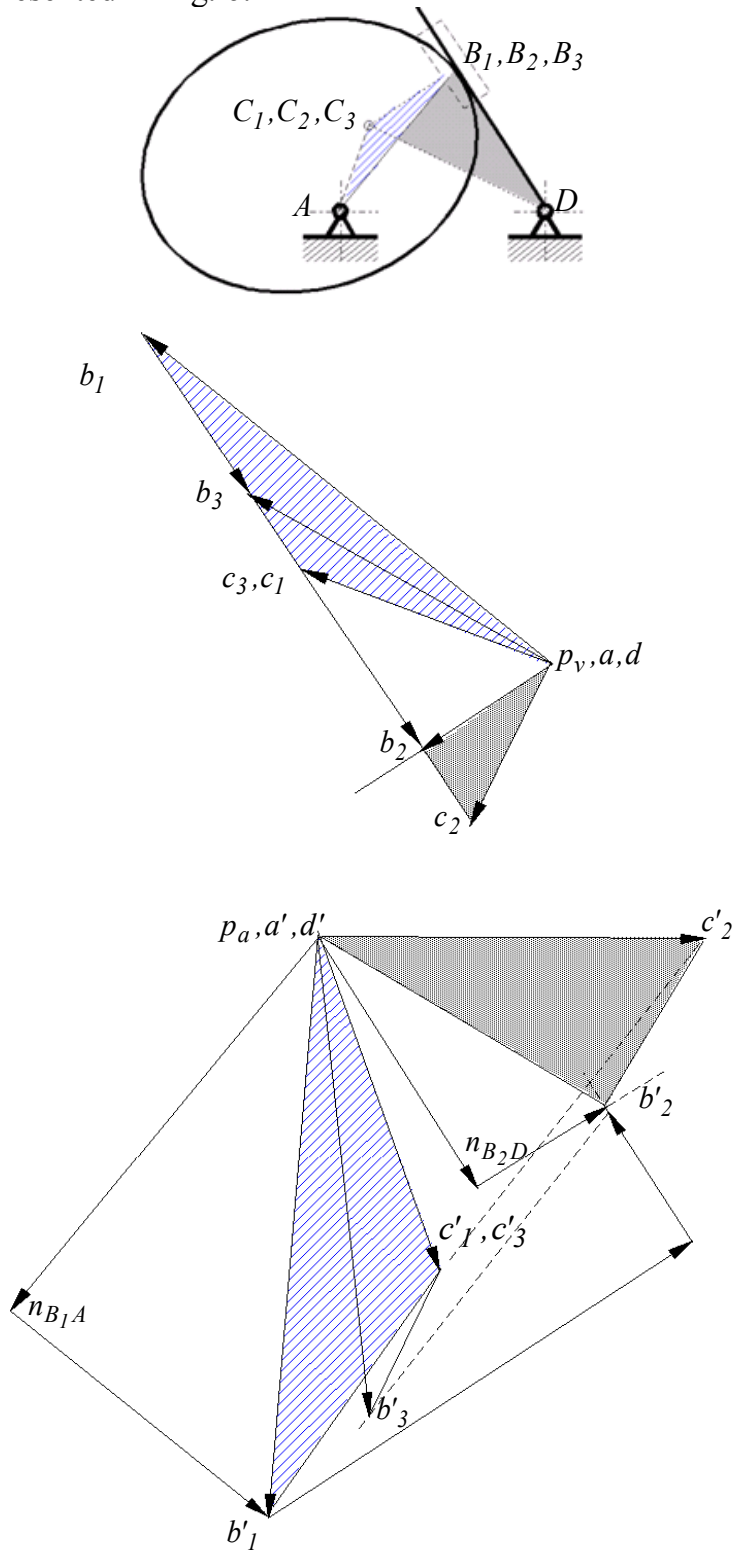

Fig. 6. The mechanisms, the velocity polygon and the accelerations polygon
The acceleration $a_{B_{2} D}^{t}$ is found and implicitly the angular acceleration $\widetilde{\varepsilon}_{2}$ together to the tangential component of relative acceleration $a_{B_{2} B_{1}}^{t}$. With the points $b_{1}{ }_{1}$ and $b_{2}{ }_{2}$ known in this polygon, the points $c^{\prime}{ }_{1}$ and $c^{\prime}{ }_{2}$ are found in a manner similar to the one applied for finding the points $c_{1}$ and $c_{2}$ from the velocities polygon. In the polygon of accelerations there are constructed the triangles $\quad a^{\prime} b_{1}{ }_{1} c_{1}^{\prime} \sim A B C_{1}$ and $d^{\prime} b_{2}{ }_{2} c_{2}{ }_{2} \sim D B C_{2}$ respectively. Finally, the point $b_{3}^{\prime}$ is found from the condition similar to the one from velocities, namely $b^{\prime}$ divides $c_{1}^{\prime} c_{2}^{\prime}$ in the same ratio as the point $B$ divides the segment $C_{1} C_{2}$ :

$$
\overline{b_{2}^{\prime} b_{3}^{\prime}}=\overline{c_{2}^{\prime} c_{3}^{\prime}}
$$

At this moment, the analysis is complete.

\section{Conclusions}

The present work proposes a graphical-analytical method for the kinematics analysis for a plane mechanism with rotating cam and oscillating flat-face follower. The principle of the method consists in considering simultaneously both the actual mechanism and the equivalent mechanisms with lower pairs. In this manner, the motion from the cam-follower joint can be decomposed into two motions of the contact point, a first motion of the contact point with respect to the profile of the cam and a second motion between the contact point and the profile of the follower. These two motions are necessary for establishing a number of components of the relative accelerations of the contact point-cam and of the contact point-follower.

This work was supported by a grant of the Romanian Ministry of Research and Innovation, CCCDI - UEFISCDI, project number PN-III-P1-1.2-PCCDI-2017-0404 / 31PCCDI / 2018 , within PNCDI III".

\section{References}

1. J. Uicker, G. Pennock, J. Shigley, Theory of Machines and Mechanisms (Oxford University Press 2010)

2. J. Angeles, C.S. Lopez-Cajun, Optimization of Cam Mechanisms (Kluwer Academic Publishers, Dordrecht 1991)

3. H. A. Rothbart, Cam Design Handbook (McGraw Hill 2004)

4. S. Alaci, F. C. Ciornei, C. F, Bull. IP Iasi, LII (LVI) 6X (2006)

5. C. Pelecudi,D. Maroș, V. Merticaru, N. Pandrea, I. Simionescu, Mechanisms, (EDP Bucureşti 1985)

6. S. Alaci, F.C. Ciornei, F. Buium, I.C. Romanu, O.T. Rusu, ACME 2018 (to be published) 